\title{
Cryogenic Stability of LTS/HTS Hybrid Conductors
}

\author{
Nagato Yanagi, Gourab Bansal, Tsutomu Hemmi, Kazuya Takahata, and Toshiyuki Mito
}

\begin{abstract}
Hybrid-type superconductors are proposed by utilizing a bundle of high-temperature superconducting (HTS) tapes as a stabilizer of low-temperature superconducting (LTS) cables in order to extend the basic research on the cryogenic stability of solid composite-type superconductors and to explore its potential. Since the effective resistivity of HTS is significantly lower than that of pure metals of equivalent cross-sectional area, a bundle of HTS tapes may work as a good stabilizer to achieve high current density. Short sample experiments have been carried out by modifying the aluminum-stabilized superconductor used for the LHD helical coils and the cryogenic stability was examined.
\end{abstract}

Index Terms-Bi-2223, cryogenic stability, HTS, hybrid conductor, pure aluminum.

\section{INTRODUCTION}

$\mathbf{L}$ ARGE current capacity with high current density is supposed to be achieved by solid composite-type low-temperature superconducting (LTS) conductors when they are extrastabilized with low resistive metals. Such an example is seen in the aluminum-stabilized superconductor used for the helical coils of the Large Helical Device (LHD) [1]. However, it was found in this conductor that the cold-end recovery current was lower than the expected value due to the enhancement of the magnetoresistivity of aluminum-copper composites by the generation of "Hall currents" under high magnetic field. A CuNi insulating layer was added as a cladding material around the pure aluminum in order to mitigate the generation of Hall currents [2], and the effective resistivity was reduced and the recovery current was increased to meet the required specification for the LHD operation point of $13 \mathrm{kA}$ at $7 \mathrm{~T}$. However, it was later found that in the transient process, the minimum propagation current of this conductor was lower than the recovery current (which can be found only in the steady-state). This is due to the long magnetic diffusion time constant in the pure aluminum ( $\sim 50 \mathrm{~ms}$ with $5 \mathrm{~N}$ purity and $6 \mathrm{~mm}$ thickness), and asymmetrical propagation velocity was found. With a transport current just above the minimum propagation current, it has been also found that the normal-zone propagates only in one direction along the conductor by forming a "traveling normal-zone" [3], [4]. Due to this fact, partial and transient normal-transitions have been observed with the LHD helical coils [5].

If these problems with composite-type LTS conductors are solved, the cryogenic stability could be sufficiently improved,

Manuscript received August 31, 2006.

N. Yanagi, T. Hemmi, K. Takahata, and T. Mito are with the National Institute for Fusion Science, 322-6 Oroshi-cho, Toki, Gifu 509-5292, Japan (e-mail: yanagi@LHD.nifs.ac.jp).

G. Bansal is with the Graduate University for Advanced Studies, 322-6 Oroshi-cho, Toki, Gifu 509-5292, Japan.

Digital Object Identifier 10.1109/TASC.2007.898172 and this type of conductors may still be used in the future middle-scale or large-scale magnets. In this connection, for the purpose of extending the basic research on the cryogenic stability of composite-type superconductors, high-temperature superconducting (HTS) wires are being examined as stabilizers in place of pure aluminum. Since HTS wires have effectively zero resistivity as long as the transport current is lower than the critical current, they can be regarded as ideal stabilizers. For the HTS, we currently use Bi-2223/Ag wires that have been developed rapidly in recent years for many applications [6], [7].

We may call this type of conductor a "LTS/HTS hybrid conductor", hereafter. Though it is not our intention to apply hybrid conductors for real magnets at the moment, one may consider a sufficiently long hybrid conductor, and its properties, especially concerning the cryogenic stability, can be examined in comparison to the conventional metal-stabilized conductors. Here, we may assume that the stabilizing HTS wires are to be used in pieces (subdivided in the longitudinal direction) so that the mechanical brittleness and relatively large AC loss generation of the HTS wires can be mitigated. In this situation, we may also assume that the transport current initially flows only in the LTS wires. When there is a normal-transition in the LTS wires due to some disturbances, the transport current may immediately transfer to the HTS, and thus, the heat generation can be suppressed and a full quench might be avoided.

We believe that hybrid conductors will explore a new research field for the cryogenic stability of LTS conductors. At the same time, they may also contribute in the development of full HTS conductors in the future.

\section{PoP EXPERIMENTS With SMALl SAMPLES}

In order to examine the concept of LTS/HTS hybrid conductors, we started by preparing small samples as the Proof-of-Principal (POP) experiment. A NbTi/Cu Rutherford cable of $3.8 \mathrm{~mm}$ by $2 \mathrm{~mm}$ size (critical current: $1360 \mathrm{~A}$ at $8 \mathrm{~T}$ ) was wound on a bobbin of $66 \mathrm{~mm}$ diameter and two and/or three Bi-2223/Ag tapes were soldered onto it to form a hybrid conductor. The cross-sectional area and current capacity are roughly ten times smaller than those of the LHD conductor. The stability tests were performed by initiating a quench from the $\mathrm{NbTi} / \mathrm{Cu}$ cable using a resistive heater attached to the conductor surface, and propagation of generated normal-zones were monitored by voltage taps. As the results are discussed in detail in [8] and [9], these hybrid conductors showed good stability and the minimum propagation currents were found to be much higher than that for an aluminum-stabilized conductor with a similar size. Thus, we considered that the concept was proved at least with small conductors. However, by increasing the conductor size, the time constant for current diffusion becomes much longer than that of heat diffusion, and the question still remains whether the minimum propagation current could 
(a)

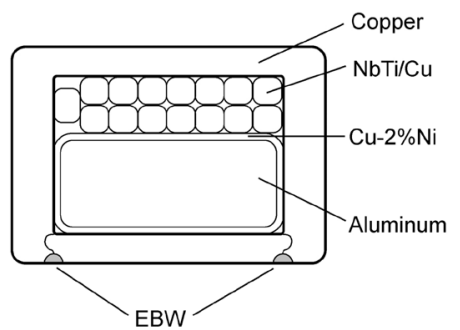

(b)

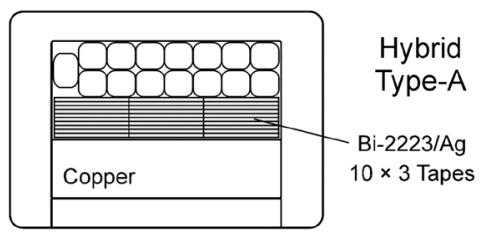

(c)

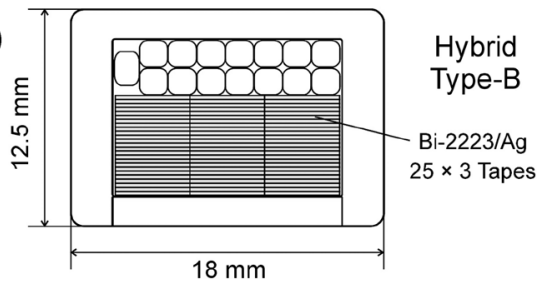

Fig. 1. Cross-sectional views of (a) the aluminum-stabilized conductor used for the LHD helical coils, (b) hybrid conductor "Type-A," and (c) hybrid conductor "Type-B."

be really improved. And thus, we fabricated bigger hybrid conductors and the stability tests were performed.

\section{EXPERIMENTAL SETUP OF Big Conductor SAMPLeS}

Fig. 1 shows the cross-sectional views of the hybrid conductors prepared and tested in the present experiments. Both conductors were fabricated by modifying the original aluminum-stabilized conductor used for the LHD helical coils. The aluminum-stabilizer was replaced by a bundle of Bi-2223/Ag tapes. We have made two types of conductors: Type-A was equipped with 30 tapes, whereas 75 for Type-B. The major specifications of these conductors are listed in Table I. For both conductors, the aluminum stabilizer was first removed from the original conductor and stacks of HTS tapes were inserted into the grooves where the aluminum stabilizer was originally situated. For each conductor, the HTS tapes were prepared with $900 \mathrm{~mm}$ length at the central region where the external magnetic field was applied, and the stacks of tapes were soldered together. The total length of each conductor was about $2 \mathrm{~m}$, and the HTS tapes were imbedded also in the end regions of the conductor samples $(\sim 500 \mathrm{~mm}$ each $)$. As there were gaps between the central HTS bundles and the other ones, the transport current could be initially supplied only to the (continuous) $\mathrm{NbTi} / \mathrm{Cu}$ cables from the current leads. For the Type-A hybrid conductor, copper strips were also used in addition to the HTS tapes in order to fill the remaining vacant space after removing the original aluminum stabilizer. Finally, the two samples were jointed together by overlapping $200 \mathrm{~mm}$ length at one end of each conductor so that a hairpin like configuration was formed. The samples were tested in the bias magnetic field simultaneously, as is illustrated in Fig. 2.
TABLE I

MAJOR SPECIFICATIONS OF HYBRID CONDUCTORS

\begin{tabular}{cll}
\hline \hline Conductors & Items & Parameters \\
\hline Original & Size & $12.5 \mathrm{~mm} \times 18 \mathrm{~mm}$ \\
Conductor & Superconductor & NbTi \\
& Strands & $\phi 1.74 \mathrm{~mm} \times 15$ \\
& Filaments & $\phi 47 \mu \mathrm{m} \times 726$ \\
& Copper to NbTi ratio & $0.9($ strand) \\
& Critical current (density) & $21 \mathrm{kA}$ at $7 \mathrm{~T}\left(1360 \mathrm{~A} / \mathrm{mm}^{2}\right)$ \\
& Resistivity of Aluminum & $3.8 \times 10^{-11} \Omega \mathrm{m}(7 \mathrm{~T}, 4.2 \mathrm{~K})$ \\
Hybrid & Surface treatment & Oxidization \\
Conductors & HTS stabilizer & Bi-2223/Ag tapes \\
& Critical current of a & $114 \mathrm{~A}$ at $77 \mathrm{~K}$, self-field \\
& single HTS tape & Type-A: 30 \\
& Number of HTS tapes & Type-B: 75 \\
& Critical current of HTS & Type-A: $14.4 \mathrm{kA}$ \\
& bundles at 4.2 K, $8 \mathrm{~T}$ & Type-B: $36 \mathrm{kA}$ \\
& (parallel field) & \\
\hline
\end{tabular}

On the conductor surfaces, a number of voltage taps were attached to monitor normal-zone development. They were situated on the narrower side of the conductor in order to minimize inductive signals due to the change of the current path in the conductors duirng normal-transitions of $\mathrm{NbTi} / \mathrm{Cu}$ cables. The voltage tap numbers are indicated in Fig. 2. In order to strictly specify the cooling perimeter and to sustain the large electromagnetic force, GFRP blocks were used as clamps. The cooling perimeter of $67 \%$ was selected in order to match the situation in the innermost windings of the LHD helical coils. Resistive heaters were attached to the conductor surfaces to initiate a quench in stability tests. In order to monitor the change of the current path during normal-transition processes, an array of Hall probes were installed around the conductors.

The fabricated short samples were then installed into the cryostat of the superconductor testing facility, which consists of $9 \mathrm{~T}$ split coils (diameter $\sim 1 \mathrm{~m}$ ), a pair of current-leads (maximum $100 \mathrm{kA}$ capacity) and $75 \mathrm{kA}$ DC power supplies. The uniform magnetic field (above 90\% level) can be applied for about $250 \mathrm{~mm}$ length at the sample centers.

\section{EXPERIMENTAL RESULTS}

Fig. 3 shows typical waveforms of the voltage tap signals observed with the Type-B hybrid conductor during a stability test. The bias magnetic field was $7 \mathrm{~T}$ and the transport current was $13.0 \mathrm{kA}$, which corresponds to the target point of the LHD helical coils. A heater pulse of $40 \mathrm{~ms}$ was injected and a normal-transition was observed. Here, only the voltage tap signals of V5, V6, V7 and V8 are plotted and we should note that the normal-zone started from the heater position of V5. We observed that the normal-zone propagation was almost symmetrical and the waveforms of V4 to V1 looked almost the same as those of V5 to V8 (and hence for simplicity they are not plotted in Fig. 3). It should be noted that the voltage waveforms indicate that the normal-zone formed a traveling normal-zone. Because of the limited length of the uniform magnetic field, the V8 


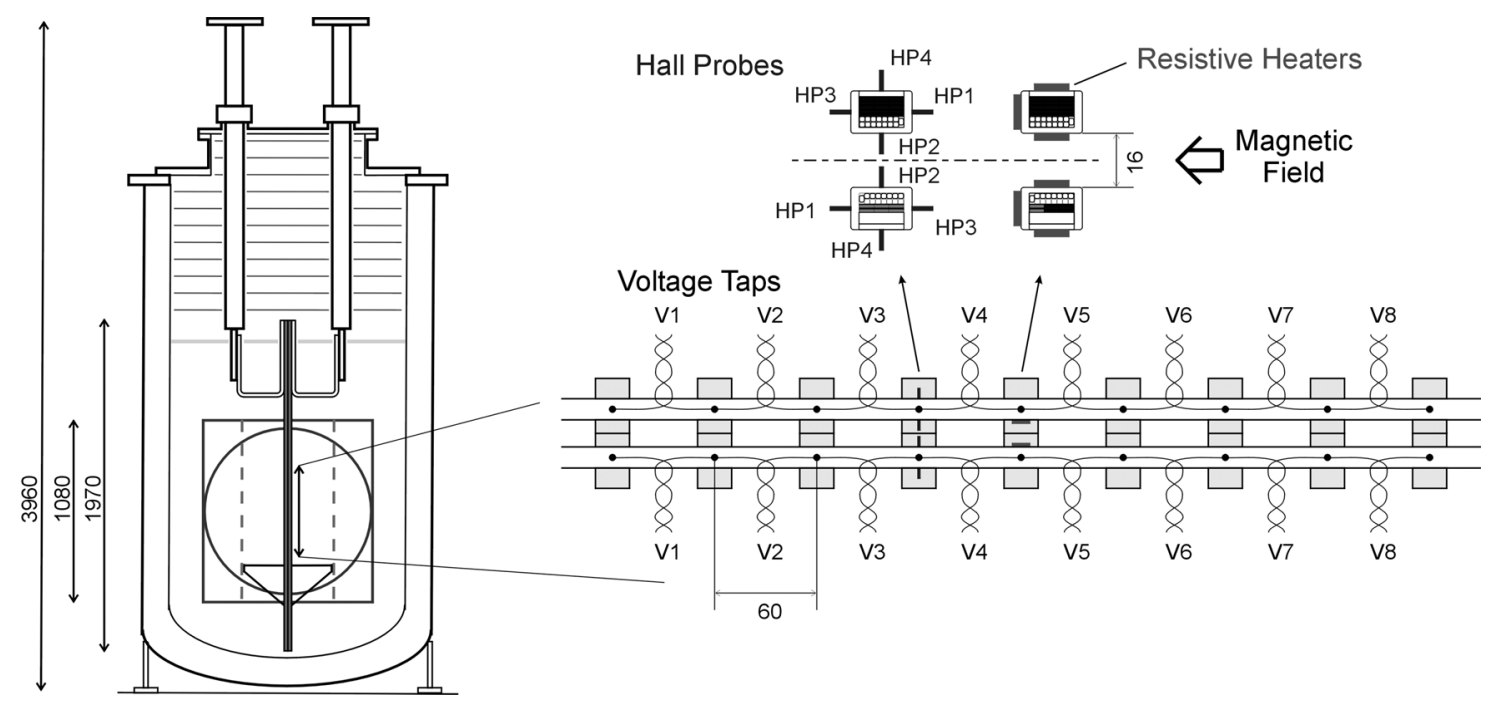

Fig. 2. Schematic illustration of the superconductor testing facility (left) and distribution of diagnostic sensors of the short samples made of two hybrid conductors (right). Unit: $\mathrm{mm}$.

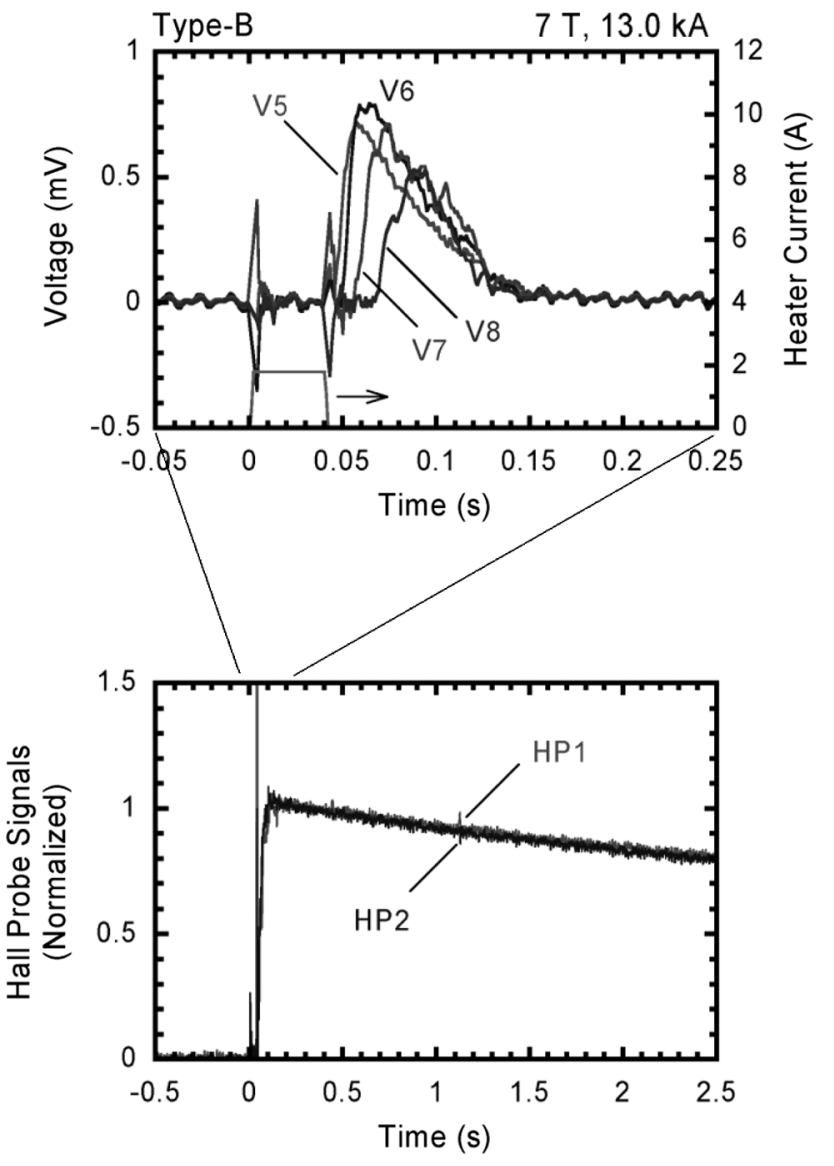

Fig. 3. Typical waveforms of the voltage tap and Hall probe signals observed at $7 \mathrm{~T}, 13 \mathrm{kA}$ for the Type-B hybrid conductor.

signal looks already a bit smaller and the profile of the traveling normal-zone is not very clear in the present test.

From the time delay between the normal-transitions at voltage tap positions between V3-V2 and V6-V7, we evaluated the propagation velocity and the result is plotted as a function of

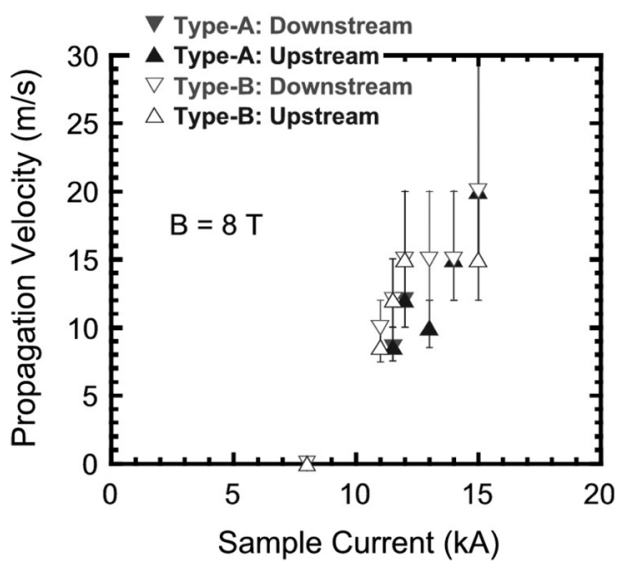

Fig. 4. Propagation velocity as a function of the sample current. "Upstream" and "Downstream" refer to the upstream and downstream directions of the sample current.

the sample current in Fig. 4. As is seen in Fig. 4, the conductor experiences normal-zone propagations even from the sample current of $\sim 8 \mathrm{kA}$. In the low current region of $8-11 \mathrm{kA}$, the normal-zone appeared only up to V3 and V6 positions, and the propagation velocity could not be properly evaluated.

As is seen in Fig. 3(b), the Hall probe signals indicate that the transport current actually transferred from the quenching $\mathrm{NbTi} / \mathrm{Cu}$ cable to the HTS stabilizer within the same time period of the voltage generation. It is also clear that after the transfer of the current, it takes a rather long time (time constant $>10 \mathrm{~s}$ ) for the transport current to come back to the $\mathrm{NbTi} / \mathrm{Cu}$ strands from the HTS bundle. The time constant should be determined by the joint resistance and inductance of the circuit formed by the $\mathrm{NbTi} / \mathrm{Cu}$ cable and the HTS stabilizer.

\section{DISCUSSION}

Though the generated normal-zone showed propagation, we may consider that the cryogenic stability of the hybrid conductors is sufficiently high. This can be confirmed by comparing 


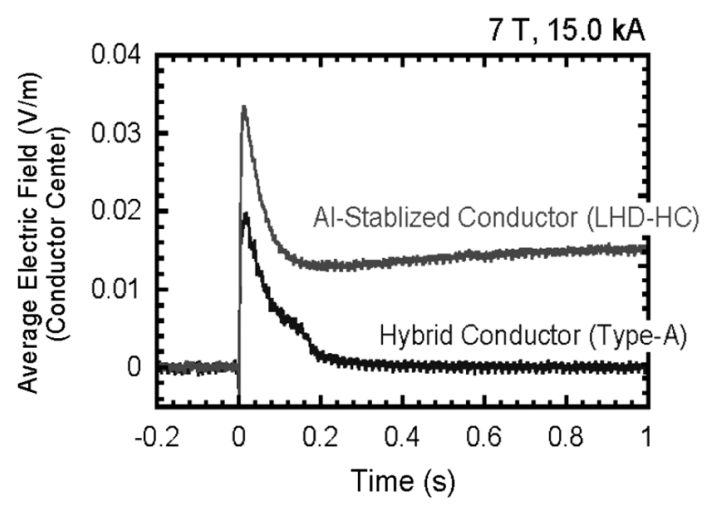

Fig. 5. Comparison of the voltage waveforms (at the heater positions) between the hybrid conductor Type-A and the original aluminum-stabilized conductor at $7 \mathrm{~T}, 15 \mathrm{kA}$.

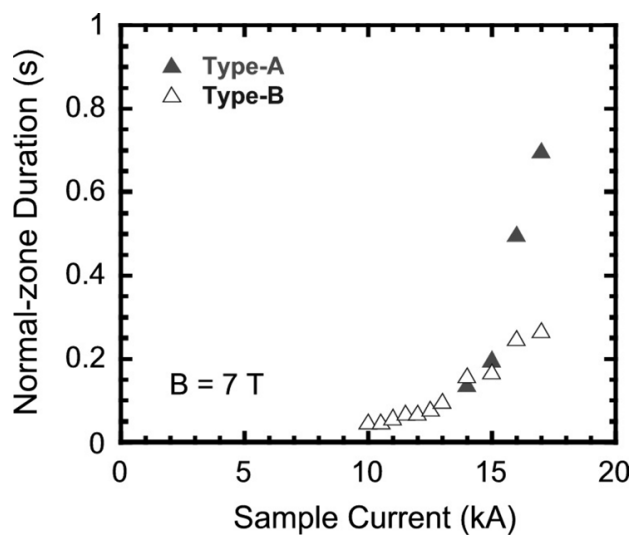

Fig. 6. Duration of the observed normal-transition as a function of the sample current.

the voltage waveforms of the hybrid conductors with those obtained with the original aluminum-stabilized conductor used for the LHD helical coils. Fig. 5 shows such an example at $8 \mathrm{~T}$, $15 \mathrm{kA}$. It was observed for the original conductor that the current of $15 \mathrm{kA}$ was well above the minimum propagation current so that the normal-zone propagated and it never disappeared even with the limited length of the uniform magnetic field. On the contrary, the hybrid conductor showed a very short transition and recovered back to the superconducting state within $0.2 \mathrm{~s}$ in this case. Fig. 6 summarizes the relationship between the duration of the observed normal-zone (at the heater position) and the sample current. It is clearly seen that the duration of the generated normal-transition is about $0.1 \mathrm{~s}$ at the current of $13 \mathrm{kA}$ (target point of the LHD conductor). Thus, we may state that hybrid conductors show good stability compared to conventional metal-stabilized conductors of the similar size.
On the other hand, regarding the mechanism of the normaltransition with the present hybrid conductors, we consider that it is caused by the current diffusion process itself from the LTS cable to the bundle of HTS stabilizer. As the transport current diffuses into the bundle, there is a change of the magnetic flux and electric field is hence generated and the joule heat is released. This is a physical process similar to flux jumping in superconducting filaments. Then, the heat release during the current transfer is unavoidable as far as a thick stabilizer is used even if its resistivity is very low. Therefore, it is strongly recommended that stabilizers should be used in pieces and not in bulk.

In Fig. 6, it is seen that when the current exceeds $15 \mathrm{kA}$, the duration of a normal-transition becomes rapidly longer for the hybrid conductor Type-A. This current may be corresponding to the critical current of $\mathrm{Bi}-2223 / \mathrm{Ag}$ cables with 30 tapes at the corresponding temperature. Though the sample was equipped with Cernox temperature sensors, they were lost during the experiment and the actual temperature of the conductors could not be measured. For the Type-B conductor, there is no such a bend in the curve of Fig. 6, since the critical current is supposed to be much higher. Due to a minor failure in the facility, the transport current could not be increased further in this experiment, and the limit of the hybrid conductors (where the HTS tapes will also experience a quench) could not be examined so far.

\section{REFERENCES}

[1] O. Motojima, K. Akaishi, and H. Chikaraishi et al., "Progress summary of LHD engineering design and construction," Nuclear Fusion, vol. 40, pp. 599-609, 2000.

[2] H. Kaneko, "Insulation of highly conductive metal in composite stabilizer for reduction of hall current across surface," Cryogenics, vol. 33, pp. 1077-1085, 1993.

[3] J. M. Pfotenhauer, M. K. Abdelsalam, and F. Bodker et al., "Test results from SMES proof of principle experiment," IEEE Trans. Magn., vol. 27, pp. 1704-1707, 1991.

[4] N. Yanagi, S. Imagawa, and Y. Hishinuma et al., "Asymmetrical normal-zone propagation observed in the aluminum-stabilized superconductor for the LHD helical coils," IEEE Trans. Appl. Supercond., vol. 14, pp. 1507-1510, 2004

[5] S. Imagawa, N. Yanagi, and H. Sekiguchi et al., "Performance of the helical coils for the large helical device in six years' operation," IEEE Trans. Appl. Supercond., vol. 14, pp. 1388-1393, 2004.

[6] L. Masur, D. Parker, M. Tanner, and E. et al., "Long length manufacturing of high performance BSCCO-2223 tape for the Detroit Edison power cable project," IEEE Trans. Appl. Supercond., vol. 11, pp. 3256-3259, 2001.

[7] T. Kaneko, T. Hikata, and M. Ueyama et al., "Status of Bi-2223 tapes performance and development," IEEE Trans. Appl. Supercond., vol. 9, pp. 2465-2468, 1999.

[8] G. Bansal, N. Yanagi, T. Hemmi, K. Takahata, and T. Mito, "Stability measurements of LTS/HTS hybrid superconductor," Fusion Eng. Des. vol. 81, pp. 2485-2489, 2006.

[9] G. Bansal, N. Yanagi, T. Hemmi, K. Takahata, and T. Mito, Experimental Investigation of the Minimum Propagation Currents and Quench Characteristics of LTS/HTS Hybrid Conductor, this conference. 